\title{
Glutamine and Glutamate Complex, as Measured by Functional Magnetic Resonance Spectroscopy, Alters During Face-Name Association Task in Patients with Mild Cognitive Impairment and Alzheimer's Disease
}

Geon-Ho Jahng, Janghoon Oh, Do-Wan Lee, Hyug-Gi Kim, Hak Young Rhee, Wonchul Shin, Jong-Woo Paik, Kyung Mi Lee, Soonchan Park, Bo-Young Choe and Chang-Woo Ryu

[Journal of Alzheimer's Disease 52(1), 2016, 145-159, DOI 10.3233/JAD-150877]

On page 150, the Figure $2 \mathrm{~b}$ had a mistake: The text $\mathrm{tCr}(\mathrm{Cr}+\mathrm{PCr})$ was switched with tCho (GPC+PCh). The correct figure is printed here:

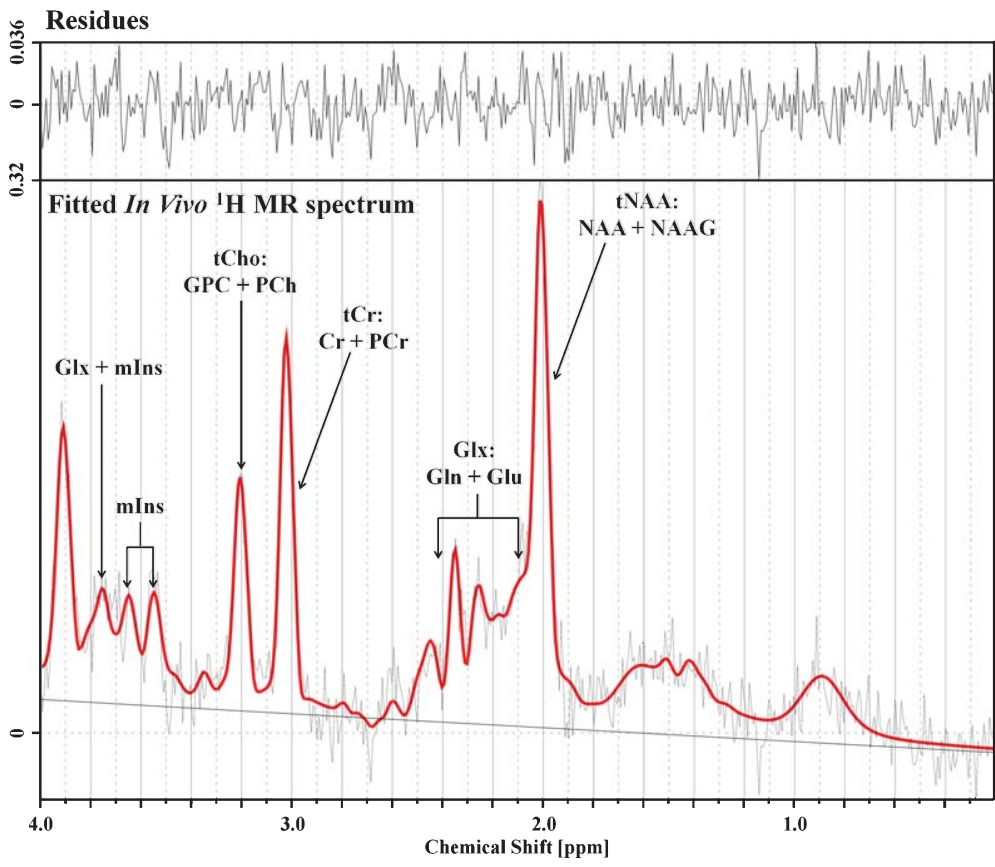

